HANNAH ZINDEL

\title{
WERKZEUG WINDKANAL
}

\section{Simulationen in der Stadtklimaforschung}

1 Vgl. BMBF (Hg.): Bekanntmachung des Bundesministeriums für Bildung und Forschung von Richtlinien zur Fördermaßnahme Stadtklima im Wandel, in: Bundesanzeiger, B4, 4.3.2015.

2 Ebd.

3 Den Begriff «Werkzeug» verwendete Prof. Dr. Bernd Leitl, stellvertretender Leiter des Meteorologischen Instituts Hamburg, während meines Besuchs des Instituts im April 2017.

$4 \mathrm{Vgl}$. Helmut Kraus: Grundlagen der Grenzschichtmeteorologie. Einführung in die Physik der atmosphärischen Grenzschicht und in die Mikrometeorologie, Berlin, Heidelberg, New York 2008, 3. Die atmosphärische Grenzschicht lagert der festen Erdoberfläche (Land, Eis) und der flüssigen (Meere, Seen) direkt auf und koppelt sie an die darüber liegende freie Atmosphäre.
$[U C]^{2}$ - Urban Climate Under Change - so lautet der Name einer Fördermaßnahme, die das deutsche Bundesministerium für Bildung und Forschung (BMBF) im Juni 2016 gestartet hat. Das Forschungsprogramm soll dazu dienen, ein «Planungswerkzeug» für eine nachhaltige Stadtplanung zu entwickeln. ${ }^{1}$ Ziel des dreijährigen Projektes ist die Entwicklung eines «leistungsstarken, innovativen und gut anwendbaren Stadtklimamodells». ${ }^{2}$ Für Städte der Größe von Stuttgart bis Berlin sollen mikroklimatische Prozesse in Computern simuliert werden. In insgesamt 30 Teilprojekten wird eine Computersimulation für urbanes Klima entwickelt, evaluiert und auf ihre Praxis- und Nutzer_innentauglichkeit getestet.

Der Aufsatz untersucht Stadtklimasimulationen aus medienhistorischer Perspektive. Konkreter liegt der Fokus auf der Frage, wie bestimmte Apparaturen, Techniken und Symbole das Wissen über Stadtklima geprägt haben. In der Meteorologie stehen drei sogenannte Werkzeuge zur Erforschung der Atmosphäre zur Verfügung: Naturmessungen, Windkanäle und Computersimulationen. ${ }^{3}$ Während im meso- und makrometeorologischen Bereich mit Computersimulationen gearbeitet wird, entzieht sich der mikrometeorologische Bereich wegen seiner hohen Komplexität und Nichtlinearität der mathematischen Modellierung und der verfügbaren Rechenleistung. Der Aufsatz konzentriert sich daher auf einen speziellen Typ Windkanal, der seit über 6o Jahren ein infrastrukturelles Rückgrat für Prozesse der Verarbeitung, Erzeugung und Validierung von Stadtklimadaten bildet: den Grenzschichtwindkanal. Anders als Windkanäle, in denen Autos oder Flugzeuge getestet werden, dienen Grenzschichtwindkanäle dazu, die atmosphärische Grenzschicht zu studieren. Das ist die unterste Schicht der Atmosphäre, deren Höhe von einigen Hundert bis zu wenigen Tausend Metern Höhe variiert. ${ }^{4}$

Die Hypothese des Aufsatzes ist, dass Stadtklima und Simulation in zweifacher Weise verbunden sind: Zum einen sind Grenzschichtwindkanäle Werkzeuge zur Verarbeitung und Erzeugung von Stadtklimadaten. Der genauere Blick auf Tests in zwei frühen Grenzschichtwindkanälen I963 und I97 I zeigt, 


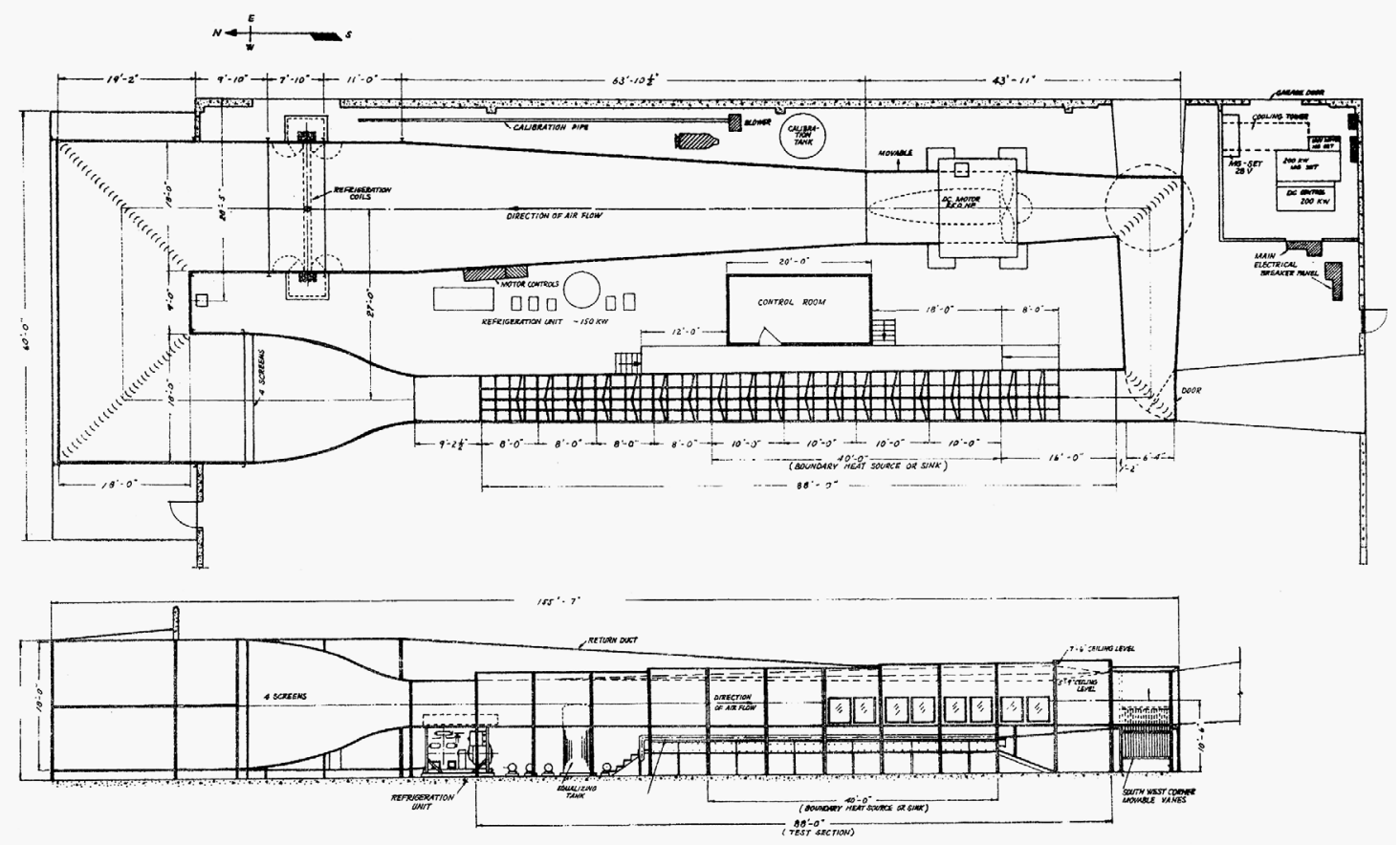

dass die Operationen zum Prozessieren und Produzieren von Stadtklimadaten sich in einer Verschränkung von meteorologischem und ingenieurtechnischem Wissen zu einer Wissenspraktik zusammenfügen, die sich als analoge Simulation bezeichnen lässt. ${ }^{5}$ Mit anderen Formen der Simulation teilt diese Wissensform eine konstitutive Ungenauigkeit; ihre Ergebnisse konfigurieren eine veränderte Form von Faktizität jenseits binärer Zuschreibungen wie 〈wahr〉 und <falsch〉. Zum anderen sind Grenzschichtwindkanäle als Instrumente des Stadtmanagements Medien der Planung und Entscheidung. Der Output aus den analogen Simulationen dient als Grundlage für die bauliche Gestaltung urbaner Flächen. Der epistemische Status analoger Simulationen implementiert sich via Grenzschichtwindkanal ab den I970er Jahren in eine zunehmend institutionalisierte Stadtklimaforschung und prägt deren Wissen über das Verhältnis von Stadt und Klima eminent mit.

\section{Micrometeorological Wind Tunnel 1963}

Einer der Orte, an denen die Geschichte des Grenzschichtwindkanals in der Stadtklimaforschung beginnt, ist die Colorado State University in Fort Collins im Jahr I963. Einer ihrer ehemaligen Absolvent_innen war Ende der I950er als frisch promovierter Ingenieur in den kleinen Ort in der Nähe von Denver zurückgekehrt und hatte am Department of Civil Engineering das

Abb. 1 Micrometeorological Wind Tunnel 1963, Grundriss und Seitenansicht

5 Am Hamburger Institut für Meteorologie wird die Forschung an den Computersimulationen von der studierten Mathematikerin Heinke Schlünzen geleitet, während die Betreuung des Windkanallabors dem studierten Ingenieur Bernd Leitl obliegt. Dieser bezeichnet die Modellierung auch als "Kunst" oder «Handwerk». 


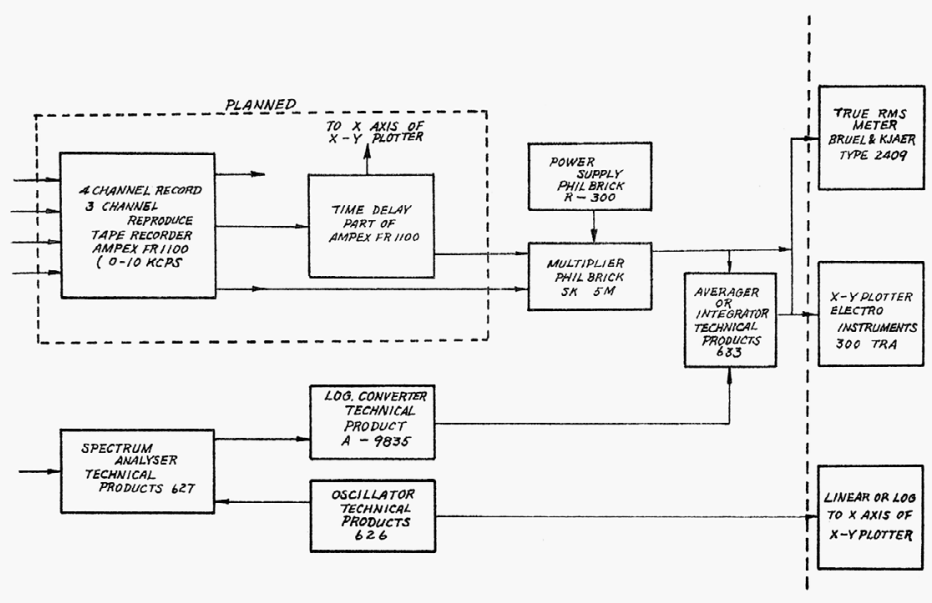

Fluid Dynamics and Diffusion Laboratory gegründet. Aufgabe dieses Labors für Strömungsdynamik und -diffusion sollte das systematische Studium der atmosphärischen Grenzschicht sein. Dazu wurde, nach verschiedenen Vorstudien, im Jahr I963 der Micrometeorological Wind Tunnel gebaut (Abb. I).

In Auftrag gegeben und finanziert wurde der Bau dieses ersten voll funktionsfähigen Grenzschichtwindkanals von der U.S. Army Electronic Research and Development Activity. Die atmosphärische Grenzschicht rückte zu

Abb. 2 Datenanalysesystem des Rechenzentrums im Micrometeorological Wind Tunnel 1963

6 Erich Plate, Jack Cermak: Micrometeorological Wind Tunnel Facility. Description and Characteristics, Abschlussbericht CER63 EJP-JEC9 Fluid Dynamics and Diffusion Laboratory, Fort Collins 1963, 1. An der Colorado State ist ausschließlich die Nutzung des Micrometeorological Wind Tunnel für zivile Forschungsfragen zugänglich dokumentiert.

7 Zur Geschichte von Wetter- und Klimaprognosen im meso- und makrometeorologischen Bereich vgl. Frederik Nebeker: Calculating the Weather. Meteorology in the 2oth Century, San Diego 1995; Paul N. Edwards: A Vast Machine. Computer Models, Climate Data, and the Politics of Global Warming, Cambridge 2010; Matthias Heymann, Gabriele Gramelsberger, Martin Mahony (Hg.): Cultures of Prediction in Atmospheric and Climate Science. Epistemic and Cultural Shifts in Computer-Based Modeling and Simulation, New York 2017.

8 Plate u. a.: Micrometeorological Wind Tunnel, 1.

9 Ebd.
Beginn der I96oer Jahre in den Fokus des wissenschaftlichen und militärischen Interesses, weil sie Schauplatz von Phänomenen war, die für die Technik- und Naturwissenschaften sowie das Militär in Zeiten des Kalten Krieges vor allem eins waren: «a problem». ${ }^{6}$ Die Unberechenbarkeit der Luftströmungen und Luftverwirbelungen nahe der Erdoberfläche, so der Forschungsbericht zum Bau des Windkanals, bedeutete eine Unkontrollierbarkeit von Raketenstarts, Radiowellen, chemischen und biologischen Substanzen, Schadstoffen und Wasserverdunstungen. Sie würden verwehen, zerstreuen und sich in unvorhersehbarer Weise verteilen. Aufgrund der wachsenden Relevanz dieser Probleme seien die bisherigen qualitativen oder phänomenologischen Beschreibungen des Strömungsfeldes nahe der Erdoberfläche nicht mehr akzeptabel. Detaillierteres Wissen sei erforderlich, das von der Erzeugung und Auswertung quantitativer Daten zu Lufttemperatur, Luftfeuchtigkeit, Luftdruck, Windgeschwindigkeit und Turbulenz in der kontrollierten Umgebung eines Windkanals zu erhoffen sei. Für den mikrometeorologischen Bereich sollte das etabliert werden, was seit den I940ern für den meso- und makrometeorologischen Bereich mit elektronischen Computern erprobt wurde: die Erstellung von Wettervorhersagen und Klimaprognosen. ${ }^{7}$ Allerdings waren und sind die kleinen Luftwirbel nahe der Erdoberfläche so hochkomplexe und nichtlineare Phänomene, dass sie sich sowohl der mathematischen Modellierung als auch der verfügbaren Rechenleistung entzogen und bis heute entziehen. In dem Forschungsbericht schlagen die Ingenieure daher vor, das fehlende «mathematical tool» mit «experimental studies» zu ersetzen. ${ }^{8}$ Dieser experimentelle Zugang sollte darin bestehen, «to simulate the earth's boundary layer in a controlled laboratory environment». ${ }^{9}$ $\mathrm{Zu}$ diesem Zweck wurde der Micrometeorological Wind Tunnel gebaut.

Die Simulation in der kontrollierten Umgebung eines Labors wurde komplett analog durchgeführt. Der Windkanal funktionierte dabei gleichermaßen 
als Datenverarbeitungs- und Datenerzeugungssystem und bildete zusammen mit anderen Einheiten des Distributions- und Diffusionslabors einen großen Analogcomputer. Die im Windkanal, dem sogenannten Data-Acquisition-System, gemessenen Werte wurden über Drähte in das separat gelegene Analog Computing Center im Tunnel Control Room (Abb. 3) geleitet. Dessen Kernstück bestand aus einem Data-Analysis System bzw. Data-Handling System (Abb. 2). Es sollte eine permanente Speicherung der eingehenden

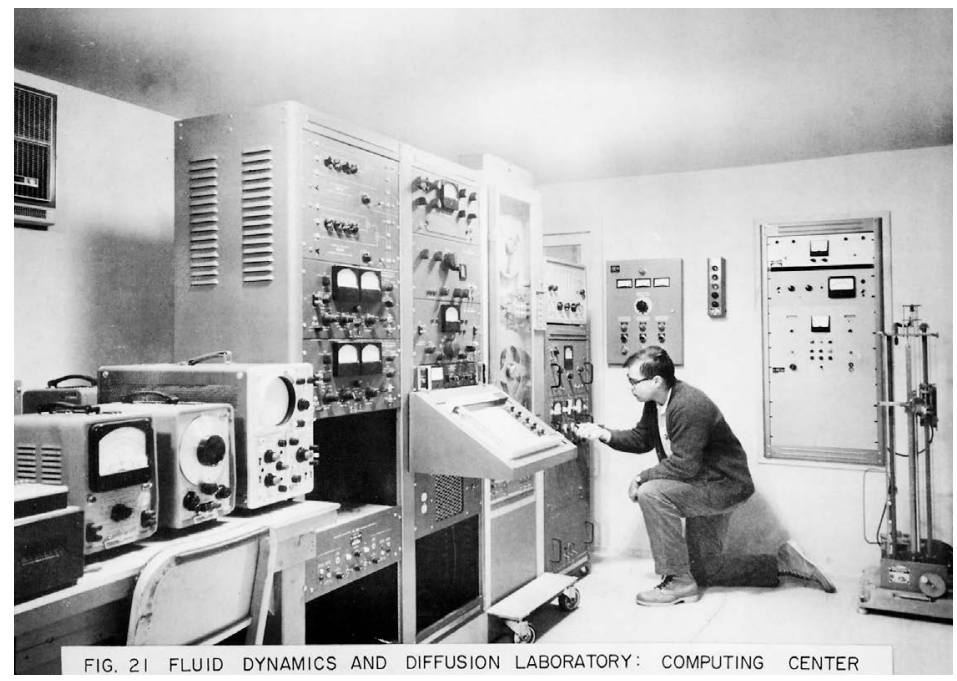
Messwerte ermöglichen (auf der Abbildung noch nicht näher spezifiziert, da - wie an der gestrichelten Linie zu erkennen - eigentlich noch in Planung), Daten aus anderen Daten herstellen (z.B. aus zwei eingehenden Signalen ein drittes ausrechnen) ${ }^{\mathbf{1 0}}$ und schließlich die eingehenden oder bereits weiter verarbeiteten Daten durch den Ausdruck auf Papier speichern. Das Data-Analysis System berechnete - in Form von Apparaten, deren von Menschenhand gesteckte Verbindungen einen Algorithmus bildeten - eine Formel, die sich auch aufschreiben ließe. Der Micrometeorological Wind Tunnel hingegen berechnete keine Formel. Er war der Ort, der konzipiert wurde, um das nicht vorhandene mathematische Werkzeug mit einem Experiment zu überbrücken.

\section{Analogcomputer Windkanal}

Dieses mathematische Werkzeug war die bis heute fehlende Theorie der Turbulenz. Verwirbelungen von Fluiden an soliden Oberflächen waren immens wichtig, um Strömungen zu verstehen, konnten aber bis zu Beginn des 20. Jahrhunderts nur auf Phänomenebene beobachtet werden." Systematische Abhilfe schuf erstmals das 1904 in Göttingen entwickelte Konzept der Grenzschicht. Der Ingenieur Ludwig Prandtl und seine Mitarbeiter_innen bauten eine Apparatur, welche die Beobachtungen von Verwirbelungen systematisierte. Sie bestand aus einem Wassertank, in dem ein handbetriebenes Schaufelrad eine regelmäßige Strömung erzeugte. In das zirkulierende Wasser wurde ein zylindrischer Körper gehalten und beobachtet, wie sich die Strömung verhielt. Separierte sich die Strömung um den Zylinder von der Gesamtströmung im Wasser, bildete sich eine verwirbelte Zone nahe der soliden Oberfläche des Zylinders, die Prandtl Grenzschicht nannte. ${ }^{12}$ In der Folge erforschte Prandtl auch turbulente Strömungen in Windkanälen. Daten, die aus Windkanälen

Abb. 3 Analoges Rechenzentrum im Fluid Dynamics and Diffusion Laboratory 1963
10 In Zeiten von Big Data würde man sagen: streambasiert-momentan wieder en vogue, um der Datenflut gerecht zu werden: direkt verarbeiten statt speichern.

11 Sichtbarmachung mit Rauch erfolgte beispielsweise in den Bewegungsstudien Étienne-Jules Mareys, vgl. Daniela Hahn: Tourbillons et turbulences. Zu einer Ästhetik des Experiments in Étienne-Jules Mareys Machines à fumée, in: Ilinx, Nr. 1, 2009, 43-69.

12 Zum Konzept der Grenzschicht, das als eine der Grundlagen der modernen Aerodynamik gilt, vgl. Ludwig Prandtl: Über Flüssigkeitsbewegungen bei sehr kleiner Reibung, in: ders., Albert Betz: Vier Abhandlungen zur Hydrodynamik und Aerodynamik, Göttingen 2010 [1904; 1927] (Göttinger Klassiker der Strömungsmechanik, Bd. 3), 1-8. Zu Prandtls Arbeit vgl. Michael Eckert: The Dawn of Fluid Dynamics. A Discipline Between Science and Technology, Weinheim 2006. 
13 Vgl. Gabriele Gramelsberger: Computerexperimente. Zum Wandel der Wissenschaft im Zeitalter des Computers, Bielefeld 2010, 74.

14 Herman H. Goldstine, John von Neumann: On the Principles of Large-Scale Computing Machines [1946], zit. n. Eric Winsberg: Science in the Age of Computer Simulation, Chicago 2010, 35. Computersimulationen kamen anfangs $v$. a. im Bereich der Ballistik und der fluid dynamics zum Einsatz, in letzterem insbesondere zur Entwicklung der Atombombe in Los Alamos, vgl. Paul Humphreys: Computer Simulations, in: Proceedings of the Biennial Meeting of the Philosophy of Science Association, Vol. 2, Chicago 1990, 497-506, hier 497.

$15 \mathrm{Vgl}$. Gramelsberger: Computerexperimente, $76-78$.

16 Vgl. Margaret Morrison: Models as Autonomous Agents, in: dies., Mary S. Morgan (Hg.): Models as Mediators. Perspectives on Natural and Social Science, Cambridge 1999, 38-65, hier 61-64.

17 vgl. ebd., 64 .

18 Robert Meroney: Ten Questions Concerning Hybrid Computational/Physical Model Simulation of Wind Flow in the Built Environment, in: Building and Environment, Vol. 96 , 2016, 12-21.

$19 \mathrm{Zu}$ analogen Simulationen mit Wasserrädern und Windkanälen siehe auch Christian Kassungs Forschungsprojekt «Kulturtechniken der analogen Simulation", dort datier 26.6.2016, leuphana.de/dfgprogramme/mecs/personen/alumni/ prof-dr-christian-kassung.html, gesehen am 2.7.2018.

20 Bernhard Dotzler: Simulation, in: Karlheinz Barck u. a. (Hg.): Ästhetische Grundbegriffe, Bd. 5, Stuttgart, Weimar 2010, 509-534, hier 509.

21 Jean Baudrillard: Simulacres et simulation, Paris 1981,12 .

22 Vgl. Humphreys: Computer Simulations; Peter Galison: Computer Simulations and the Trading Zone, in: ders., David J. Stump (Hg.): The Disunity of Science. Boundaries, Contexts, and Power, Stanford 1996, 118-157; Morrison u. a. (Hg.): Models as Mediators. abgeleitet wurden, galten allerdings zunehmend als unzuverlässig und ungenau. ${ }^{13}$ Für John von Neumann, einen der Pioniere der Computersimulation, stellten Windkanäle daher eine seltsame Art des Experimentierens dar, die nicht der Verifizierung theoretischer Annahmen diente, sondern «to replace computation from an unquestioned theory by direct measurement. Thus wind tunnels are [...] computing devices of the so called analogy type». ${ }^{14}$ Gabriele Gramelsberger hält fest, dass diese Ungenauigkeit von Windkanälen für von Neumann ein wesentlicher Motivator für den Entwurf der grundlegenden Architektur moderner Computer gewesen sei, verbunden mit der Idee, nicht mehr (ungenau) mit Experimenten zu rechnen, sondern mit Rechnern zu experimentieren. ${ }^{15}$ Margaret Morrison hebt hervor, dass Prandtl aus den WassertankBeobachtungen heraus den mathematischen Ansatz der Grenzschichttheorie formulieren konnte, und betont damit vor allem die Produktivität seines $\mathrm{Zu}$ gangs. Den Wassertank bezeichnet sie als ein Modell, das sich einerseits separat von Theorie oder Experiment befand und andererseits in beide einhaken konnte. ${ }^{16}$ Zwischen konkreten Modellen wie dem Wassertank oder abstrakteren Modellen wie Computersimulationen sieht sie keinen Unterschied. Beide würden zeigen, wie partikuläre Stücke eines Systems integriert und zusammengefügt würden in einer Weise, dass das Systemverhalten erklärt werden könne. ${ }^{17}$ Der Ingenieur Robert Meroney, Nachfolger des Gründers des Fluid Dynamics and Diffusion Laboratory an der Colorado State University, betont sogar, dass «wind or water tunnels» als «analog computers» den Vorteil einer «near-infinitesimal resolution and near-infinite memory» hätten. ${ }^{18}$ Für die weitere Betrachtung von Grenzschichtwindkanälen in der Stadtklimaforschung sei vor dem Hintergrund der hier angeführten Aspekte festgehalten: Wenn Grenzschichtwindkanäle als Analogcomputer bezeichnet werden und die Operationen darin als Simulationen, dann lässt sich diese spezifische Wissensform als analoge Simulation fassen. ${ }^{19}$ Dass die Ingenieure in dem Forschungsbericht zum Bau des Micrometeorological Wind Tunnels selbst von Simulationen sprechen, verweist zudem auf eine spezifische (Nicht-)Faktizität ihrer Daten.

Im Wörterbuch Ästhetischer Grundbegriffe fasst der Medienwissenschaftler Bernhard Dotzler den epistemischen Kern von Simulationen wie folgt zusammen: «Simulationen [lassen] Phänomene und Situationen entstehen, die keinen anderen Rückhalt haben als das Medium, das sie hervorbringt». ${ }^{20}$ Während Jean Baudrillard in den I980er Jahren deshalb beklagte, «la simulation remet en cause la différence $\mathrm{du}\langle\mathrm{vrai}\rangle$ et $\mathrm{du}\left\langle\mathrm{faux}\right.$, du 〈réel〉 et de l'<imaginaire»», ${ }^{21}$ sahen einzelne Vertreter_innen aus Wissenschaftsphilosophie und Wissenschaftsgeschichte in dem Unterlaufen solcher Dualismen gerade das Potenzial von Simulationen. ${ }^{22}$ Im Anschluss an diese spricht Claus Pias von einer $\ll \mathrm{Me}-$ thode der Simulation», die gegenüber den mathematisierten exakten Wissenschaften durch eine «prinzipielle Nicht-Exaktheit» gekennzeichnet sei, mit der sie zugleich den Horizont der behandelbaren Probleme erweitere. ${ }^{23}$ Für die analoge Simulation sei vorerst festgehalten, dass sie Klimadaten produziert, die 


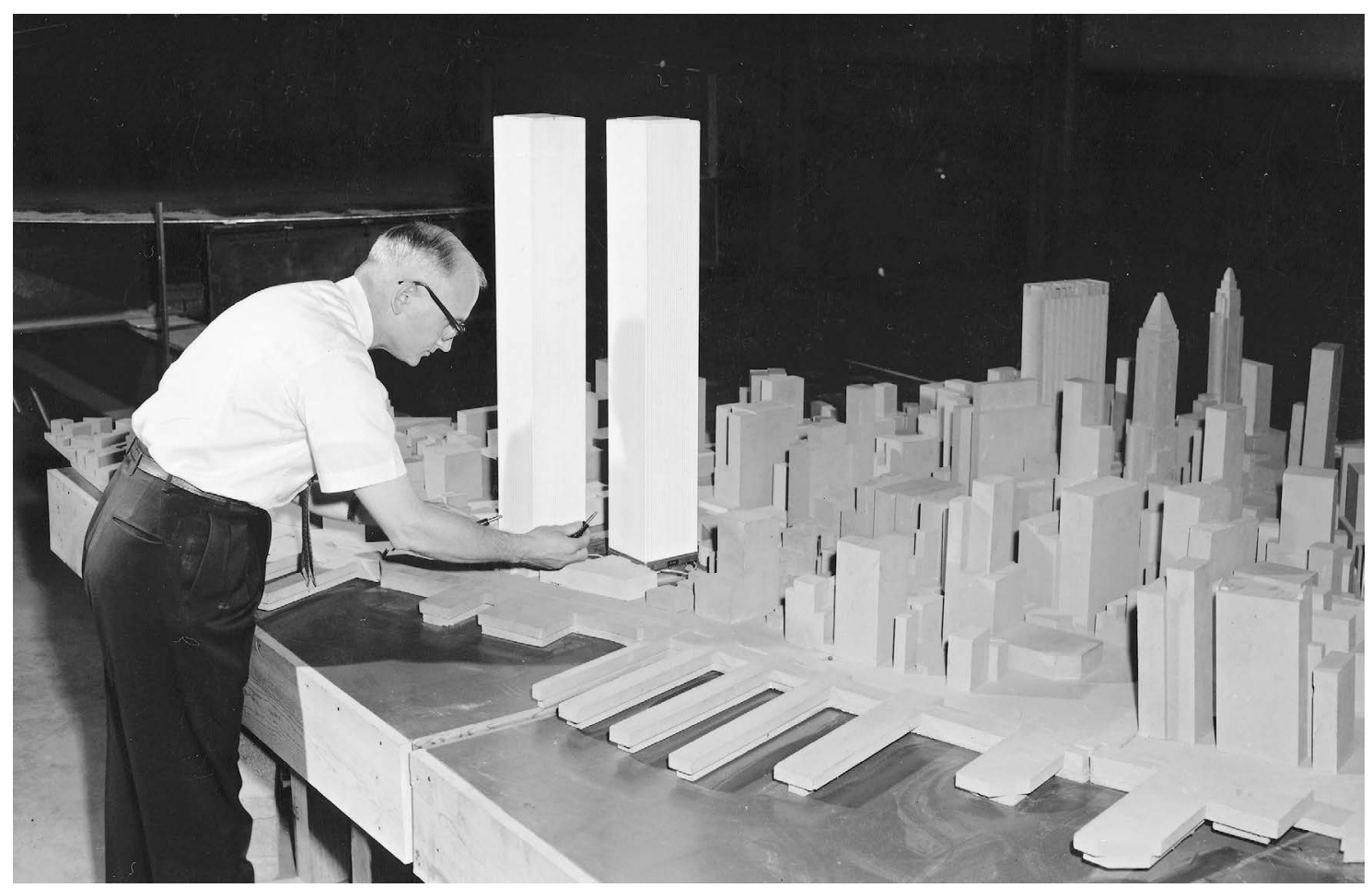

keinen anderen Rückhalt haben als die Versammlung von Dingen und Operationen, die diese hervorbringen. Gerade deswegen sind Grenzschichtwindkanäle nicht nur Werkzeuge, sondern Medien. Mit dem Grenzschichtwindkanal ist es wie mit dem Fernrohr: Wenn Galilei es in die Hand nimmt, wechselt es den Charakter, «entlässt [...] Daten ganz eigener Art und ist [...] von einem Instrument zu einem Medium geworden», es «vollzieht eine Welterzeugung durch die Einrichtung einer konstitutiven Selbstreferenz». ${ }^{24} \mathrm{Um}$ zu verstehen, von welchen $<$ Daten $>$ und <Welten $>$ die Rede sein könnte und wie sie ineinander verschränkt sind, liegt der Fokus im Folgenden auf einer der ersten Simulationen von urbanem Klima in einem Grenzschichtwindkanal.

\section{Environmental Wind Tunnel 1971}

Im Laufe der I96oer Jahre wurde in dem Micrometeorological Wind Tunnel vor allem die Windlast auf Bauten erforscht. Insbesondere der zunehmende Bau von Wolkenkratzern erforderte Vorhersagen über deren Stabilität bei Stürmen. Frühe Forschungsaufträge betrafen den Bau der Twin Towers des World Trade Centers in New York oder des Headquarter Building der Bank of America in San Francisco (Abb. 4). Ende der ig6oer Jahre kamen Recherchen zu
Abb. 4 Windkanalmodell der Twin Towers 1965

23 Claus Pias: Zur Epistemologie der Computersimulation, in: Peter Berz, Marianne Kubaczek, Eva Laquièze-Waniek, David Unterholzner (Hg.): Spielregeln. 25 Aufstellungen, 41-6o, hier 45 .

24 Joseph Vogl: Medien-Werden. Galileis Fernrohr, in: Archiu für Mediengeschichte, Nr. 1, 2001: Mediale Historiographien, 115-123, hier $115,118$. 
25 Fazal Chaudhry, Jack Cermak: Wind-Tunnel Modeling of Flow and Diffusion over an Urban Complex, Technischer Bericht CER70-7IFHCJEC24, Fluid Dynamics and Diffusion Laboratory, Fort Collins 1971, ii f. 26 Vgl. ebd., 1.

27 Zu Wissenschaften im Kalten Krieg vgl. Naomi Oreskes ( $\mathrm{Hg}$.): Science and Technology in the Global Cold War, Cambridge 2014. Spezifischer zum atomic age in urbanen Gebieten vgl. Steven Hanna: A History of Classic Atmospheric Dispersion Field Experiments, Konferenzbeitrag AMS Annual Meeting on Historical Atmospheric Dispersion Models 2010; Eva Schauerte: Dispersion. Stadtplanung zwischen Utopie und Dystopie im ‘Atomic Age), in: Zeitschrift für Medienwissenschaft, Nr. 14, 2016, 121-133.

28 Zur Feldstudie vgl. I. R. Graham: An Analysis of Turbulence Statistics at Fort Wayne, Indiana, in: Journal of Applied Meteorology, Nr. 7, 1968, 90-93.

29 Der Hitzeinseleffekt war ein bereits seit dem 19. Jahrhundert bekanntes Phänomen. Vgl. Michael Hebbert, Fionn MacKillop: Urban Climatology Applied to Urban Planning. A Postwar Knowledge Circulation Failure, in: International Journal of Urban and Regional Research, Vol. 37, 2013, 1542-1558, hier 1544 .
Inseln hinzu, die als Raketenstandpunkte interessant wurden, sowie zum Bau von Atomkraftwerken, Kohlekraftwerken und Ölraffinerien. Anfang der i 97oer Jahre verschob sich der Fokus der Forschung im Fluid Dynamics and Diffusion Laboratory an der Colorado State von großen Gebäuden auf unregelmäßige Oberflächen wie Berge, Wälder und Städte. I97 I wurde deshalb ein weiterer Windkanal gebaut, der es ermöglichen sollte, größere Flächen zu modellieren und Phänomene von Wetter und Klima über komplexem Terrain zu studieren. In dem im gleichen Jahr fertiggestellten Bericht Wind-Tunnel Modeling of Flow and Diffusion over an Urban Complex von Jack Cermak und Fazal Chaudhry werden die Ergebnisse der ersten größeren Studie in diesem sogenannten Environmental Wind Tunnel zusammengefasst.

The results of this study have proved that it is indeed possible to simulate the flow over a city and obtain useful information, relatively inexpensively, on urban diffusion. The investigation opens the way for studies of air-pollution problems for purposes of urban planning. The location of industrial sites relative to major topographical features, the location of freeways through existing cities, the grouping of tall buildings in an urban-development program, or even the judicious placing of parks, residential and industrial areas in an entirely new city to minimize air pollution potentials under adverse meteorological conditions can be studied systematically. ${ }^{25}$

Der Environmental Wind Tunnel sollte die Strömung über Städten simulieren. Auf Basis dieser Studien sollten Empfehlungen zu der Platzierung von Industrieanlagen, Highways, hohen Häusergruppen, Wohngegenden und Parks ausgesprochen werden. Der Environmental Wind Tunnel sollte nicht nur dazu dienen, Luftverschmutzung in Städten besser zu verstehen, sondern auch, diese vorherzusagen und zu kontrollieren. ${ }^{26}$ Der Bau des neuen Umweltwindkanals wurde durch das Office for Naval Research des US-Verteidigungsministeriums finanziert. Ein erhöhtes Interesse an einem besseren Verständnis der Ausbreitung von chemischen Stoffen in der Luft über Wäldern wird seitens der USA nicht zuletzt durch den Einsatz des Entlaubungsmittels Agent Orange im Vietnamkrieg bestanden haben. Nach dem zweiten Weltkrieg und Hiroshima gab es außerdem einen zunehmenden Bedarf an Wissen über die Ausbreitung von radioaktiven Stoffen aus Atombomben und Atomkraftwerken, insbesondere in urbanen Gebieten. ${ }^{27}$

Studien zu Charakteristika von Luftströmungen und Luftverwirbelungen über komplexem Terrain gab es Anfang der i970er Jahre noch relativ wenige. Eine der umfangreichsten war in den Jahren I964 bis I966 in der kleinen Stadt Fort Wayne, Indiana, im Rahmen eines großangelegten Feldexperiments durchgeführt worden.$^{28} \mathrm{Im}$ Fokus der Untersuchungsreihe hatte der Effekt sogenannter Hitzeinseln, heat-islands, gestanden, der in Studien der I 96oer Jahre herausgearbeitet worden war und Städte als Temperaturanomalien an der Erdoberfläche beschrieb. ${ }^{29}{ }_{2}$ I Messreihen mit meteorologischen Messstationen waren auf dem Boden, auf hohen Türmen sowie in Ballons durchgeführt worden. Diese Feldexperimente, kommentiert der Windkanalbericht, würden das umfänglichste 
verfügbare «set of data» ${ }^{30}$ über meteorologische Diffusion im urbanen Raum bereitstellen. Allerdings seien mehr experimentelle Untersuchungen notwendig, um Auskunft über den Einfluss urbaner Komplexe auf die Atmosphäre zu geben. Da diese teuer seien, biete der Environmental Wind Tunnel eine Alternative. ${ }^{31}$ Die Feldstudie in Fort Wayne wird zwar als rhetorische Negativfolie zur Betonung der Relevanz der eigenen Forschung verwendet, dient aber während der Experimente im Windkanal als Referenzdatensatz.

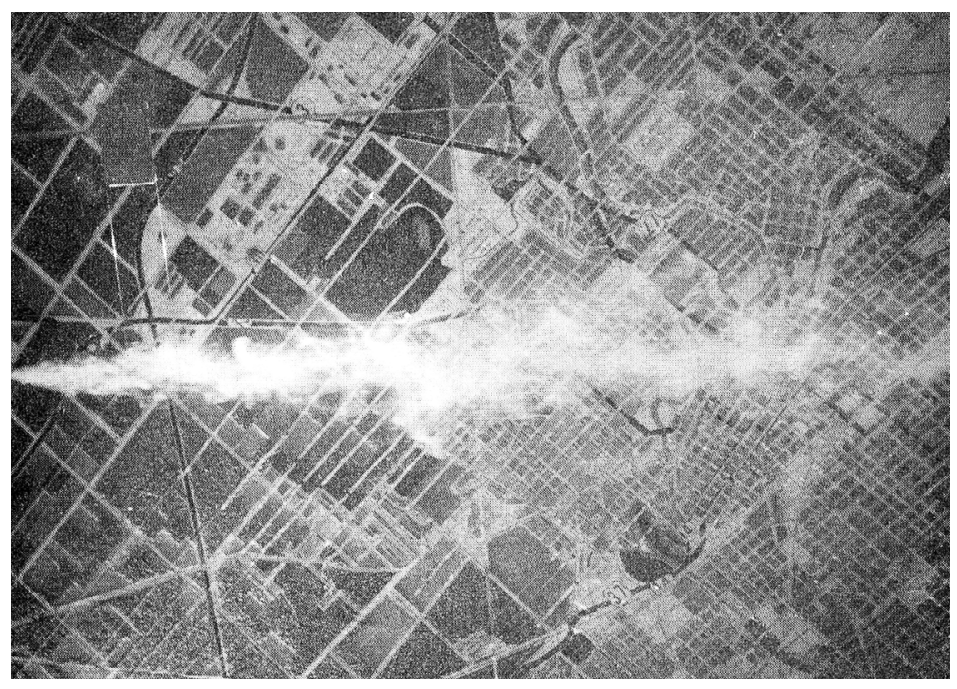
Daher lohnt sich ein genauerer Blick auf die einzelnen Operationen zur Verarbeitung und Erzeugung von Daten mit dem Environmental Wind Tunnel und zu den Praktiken des Vergleichens dieser Daten mit jenen aus der Feldstudie.

Zuerst wurde das Stadtmodell gebaut. Dazu wurde ein vergrößerter Stadtplan von Fort Wayne in die Testsektion des Windkanals geklebt. Auf diesem konnten Gebäudegruppen angeordnet werden, die aus Steinplatten gesägt waren. Gebäude mit weniger als vier Stockwerken wurden vereinfacht und als ganzer «city-block» ${ }^{32}$ modelliert, Häuser und Bäume wurden als ineinander verwoben betrachtet und ebenfalls als zusammenhängender Block gesägt. Details für hohe Gebäude entnahmen die Forscher_innen Luftfotografien. Der horizontale Maßstab des Stadtmodells betrug I:4000, der vertikale I:2000. Der Bereich zwischen Turbine und Stadtmodell diente der Vorverwirbelung des Windes. Um die nötige Oberflächenrauigkeit zu erzeugen, wurden Sperrholzplatten ausgelegt, die mit Reiskörnern beklebt waren. Die ländliche Umgebung der Stadt fand sich im Windkanal in Form einer mit Sandpapier beklebten Fläche vor dem Stadtmodell. ${ }^{33}$ Ein kleines Rohr, aus dem das leicht radioaktive und daher gut messbare Gas Krypton-8 5 strömte, ersetzte das Flugzeug, das im Feldexperiment Pigmente über der Stadt verteilt hatte. Um aus dem Stadtmodell im Windkanal ebenfalls eine Hitzeinsel zu machen, wurden Heizquellen in Form von vier langen, einzeln heizbaren Drähten über dem Modell platziert. Streifen aus Glasfasergewebe zwischen Drähten und Steinplatten schützten das Modell vor dem Verkohlen. ${ }^{34}$ Die Modellierung von Fort Wayne im Environmental Wind Tunnel war ein groBes Skalierungsprojekt, das zahlreiche Medien der räumlichen Skalierung involvierte - von kopierten Stadtplänen und Luftfotografien über gesägte Steinhäuser bis hin zu geklebten Sandpapier-Reis-Landschaften. Ebenfalls kleiner als in Feldmessungen waren die in dem Windkanal verteilten Messinstrumente. Die verwendeten Pitot-Rohre, Wärmefühler und Hitzdraht-Anemometer waren

Abb. 5 Modell von Fort Wayne während der Simulation im Environmental Wind Tunnel 1971
30 Chaudhry u. a.: Wind Tunnel Modeling, 2.

31 Vgl. ebd., $2 \mathrm{f}$.

32 Ebd., 11.

33 Wie ich bei meinem Besuch im Meteorologischen Institut Hamburg gelernt habe, kommen mittlerweile nicht mehr Sandpapier und Reis als Turbulenzgeneratoren zum Einsatz, sondern Metallwinkel und rote Legosteine.

34 Zum Aufbau des Modells vgl. Chaudhry u. a.: Wind Tunnel Modeling, 9-12. 

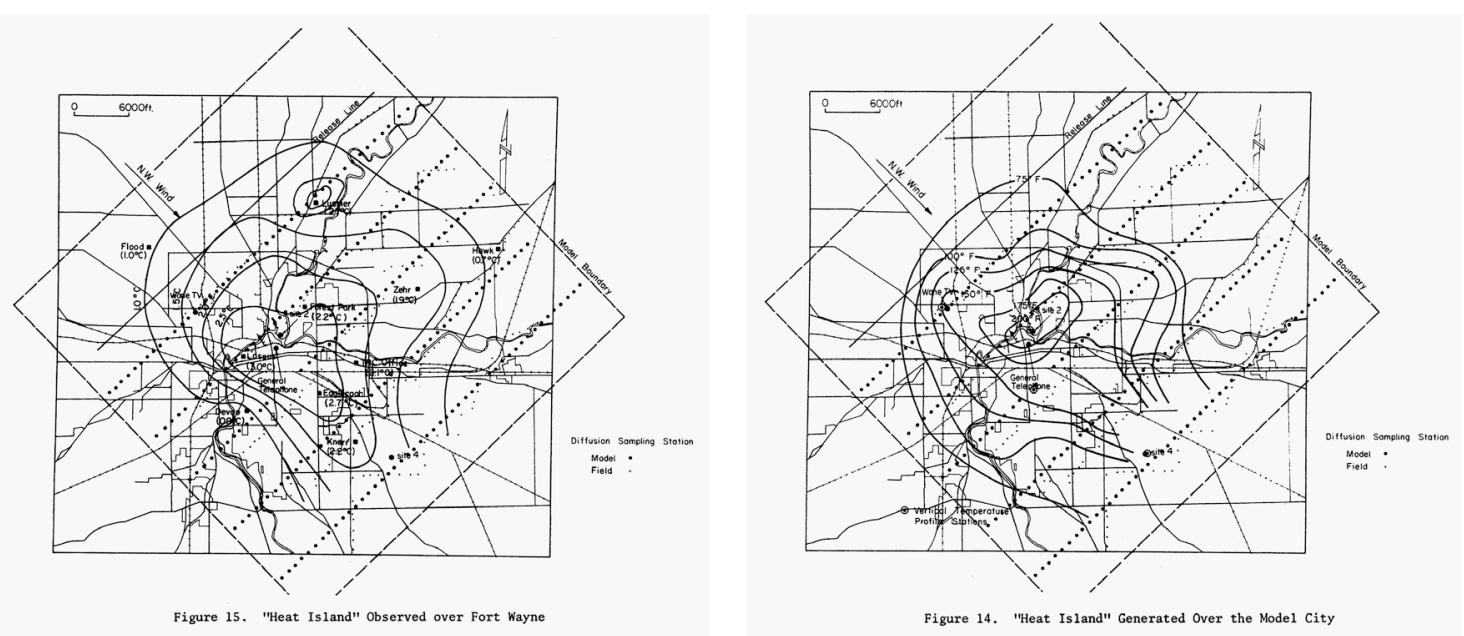

Abb. 6 Hitzeinsel über Fort Wayne aus Feldstudien-Daten

Abb. 7 Hitzeinsel über dem Modell von Fort Wayne aus den Windkanal-Daten
$35 \mathrm{Zu}$ den Instrumenten vgl. Chaudhry u. a.: Wind Tunnel Modeling, 5-7.

36 Vgl. ebd., 26. so fein, wie es technisch möglich war. Die Dichte des Krypton-85 erfasste ein Geiger-Müller-Zähler. Rauch sollte Verteilungsmuster über dem Modell sichtbar machen und wurde mit Polaroidkameras fotografiert (Abb. 5) ${ }^{35}$

Der nächste Schritt bestand in der Modellierung der Luft von Fort Wayne und Umgebung. Dazu wurden die heizbaren Drähte so kalibriert, dass die Temperatur der gesägten Steinhäuser jener Temperatur entsprach, die zu Beginn einer der Messreihen in Fort Wayne gemessen worden war. Die Turbinen des Windkanals wurden so eingestellt, dass die Windgeschwindigkeit im Windkanal jener im Feld entsprach etc. Waren diese und/oder weitere Anfangswerte kalibriert, begann die Simulation. Für einen festgelegten Zeitraum wurden die Veränderungen von Temperatur, Windgeschwindigkeit und/oder weiteren Variablen gemessen, die in der dynamischen Modellatmosphäre durch das $\mathrm{Zu}$ sammenspiel der statischen Modellstadt, der Windkanaltechnik und der Windkanalarchitektur entstanden. Da die größere räumliche Skalierung des Stadtmodells r:400o betrug, wurde auch die Dauer der Messung im Feld skaliert: Alles lief viel schneller ab. Während der Simulation wurden Veränderungen der Windgeschwindigkeit und Temperatur und ggf. weitere Werte dokumentiert, indem verschiedene Messinstrumente in ihrer je spezifischen Weise Spannungen erzeugten, die über Drähte in das Datenverarbeitungszentrum geleitet wurden und deren Stärke dort, nach einem Zwischenschritt oder direkt, auf Papier registriert wurden.

In einem letzten Schritt übersetzten die Forscher_innen die verschiedenen auf Papier gespeicherten Messwerte aus der Windkanalsimulation in Tabellen, Grafen und v.a. Landkarten. ${ }^{36}$ Mit Methoden der synoptischen Meteorologie wurden beispielsweise Isothermen, also Linien gleicher Temperatur, in einen weiteren kopierten Stadtplan von Fort Wayne eingezeichnet und neben einen Stadtplan gelegt, in den die Isothermen aus dem Feldexperiment eingezeichnet waren (Abb. 6, Abb. 7). Ihr visueller Vergleich durch eine_n Forscher_in führte 
dann zu Aussagen wie: «the rough picture of the heat island that emerges [...] is remarkably similar to that obtained in the wind tunnel». ${ }^{37}$

Die analoge Simulation in dem Environmental Wind Tunnel, so lässt sich zusammenfassen, bestand aus einem Gemenge zahlreicher Praktiken und Techniken. Der Bericht weist darauf hin, dass nie eine gleichzeitige Kalibrierung aller im Feld gemessenen Variablen möglich gewesen sei und daher nur «partielle Simulationen» ${ }^{38}$ durchgeführt wurden. Die Messergebnisse aus diesen partiellen Simulationen, im Bericht «model-data», wurden mit den Messergebnissen aus den sogenannten Feldexperimenten oder Naturmessungen verglichen, im Bericht «proto-type data». Dieser Vergleich bestand in einem Über- und Nebeneinan-

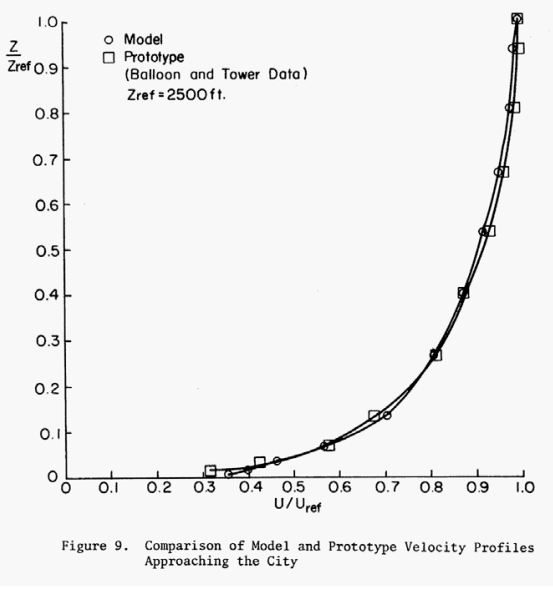
derlegen der in Tabellen, Grafen und v.a. Stadtplänen visualisierten Datensätze aus Stadt und Modellstadt (Abb. 8). In dem Experimentierrechnen verschoben sich die Verfahren zur Verifikation und Falsifikation traditioneller theoretischer und experimenteller Ansätze zu einer Suche nach «Ähnlichkeiten», «Korrespondenzen» oder «Äquivalenzen»- es ging um das Erkennen von «Mustern».39 Während das Modell der Stadt maßstabsgetreu skaliert wurde, war dies für die räumlich-zeitliche Skalierung der Atmosphäre über Fort Wayne nicht möglich. Hierin bestand und besteht eine zentrale Herausforderung von Windkanalsimulationen, die einen wesentlichen Unterschied zu Computersimulationen bildet. Bereits in dem Bericht über den Micrometeorological Wind Tunnel von 1963 heißt es: «The main shortcomings of wind-tunnel applications to micrometeorology are the requirement that the atmosphere must be scaled down while scaling laws for turbulent flow fields are not fully understood $\gg .{ }^{40}$ Der Meteorologe Michael Schatzmann hält 34 Jahre später fest, dass der Vergleich von «measured data» und «model results» ein Vergleich von «apples with oranges» sei. ${ }^{41}$

Dies hängt nicht zuletzt damit zusammen, dass die beschriebene Kette von Operationen im Environmental Wind Tunnel nicht reversibel war. ${ }^{42}$ Während Prototypdaten zu Modelldaten weiterverarbeitet werden konnten, ging dies umgekehrt nicht. Was nach der Kalibrierung während der Simulation ablief, erzeugte einen aus der restlichen Operationskette losgelösten und doch mit dieser verwobenen Raum, der sich in Anlehnung an den Namen der beiden Windkanäle der Colorado State als eigenes Mikroklima oder Environment bezeichnen lässt, innerhalb dessen eine Versammlung von Dingen wie Steinplatten, Sandpapier, Reiskörnern, Sperrholzplatten, Turbinen, heißen Drähten, Glasfaserstreifen, Pitot-Rohren, Wärmefühlern, Hitzdraht-Anemometern, Geiger-Müller-Zählern, Gasen und Windkanalarchitekturen aus Wänden, Türen, Fenstern und Kabelöffnungen zu einem teilautonomen und Evidenzen erzeugenden Kollektiv wurde.

Abb. 8 Prototypdaten aus der Feldstudie und Modelldaten aus der Windkanalstudie

37 Ebd., 27.

38 Ebd., 4.

39 Ebd., 26. Im Original: "similarities", "correspondences", «equivalences", "pattern".

40 Plate u.a.: Micrometeorological Wind Tunnel, 1.

41 Michael Schatzmann, Stilianos Rafailidis, Michel Pavageau: Some Remarks on the Validation of Small-Scale Dispersion Models with Field and Laboratory Data, in: Journal of Wind Engineering and Industrial Aerodynamics, Nr. 67/68, 1997, 855-893, hier 887.

42 Diese fehlende Reversibilität ist auch für Computersimulationen beschrieben worden. Die in diesem Zusammenhang bemühte Metapher der Simulationspipeline wird z. B. erklärt bei Petra Gehring, Christoph Hubig, Andreas Kaminski: Was ist ein Hochleistungsrechner?, in: dies. (Hg.): Computersimulationen verstehen. Ein Toolkit für interdisziplinär Forschende aus den Geistes- und Sozialwissenschaften, dort datiert 13.3.2017, tuprints.ulb.tu-darmstadt.de/6079/1/ toolkit.pdf, gesehen am 1.11.2017. 
43 Für Environments in Kontexten des Ubiquitous Computing bzw. an der Schnittstelle von Architektur und Medien werden nicht unähnliche Charakteristika der Verschränkung beschrieben und in ihren ökologischen Traditionslinien berücksichtigt. Vgl. Florian Sprenger: Architekturen des «Environment). Reyner Banham und das dritte Maschinenzeitalter, in: Zeitschrift für Medienwissenschaft, Nr. 12, 2015, 55-67. Zur Begriffsgeschichte von Environment vgl. ders.: Zwischen ¿Umwelts und ‘milieus. Zur Begriffsgeschichte von renvironments in der Evolutionstheorie, in: Forum Interdisziplinäre Begriffsgeschichte, Nr. 3, 2014, 7-18.

44 Er ist also nicht Abbild eines Urbildes, sondern Abbild eines Abbildes. Zum platonischen Motiv und der Umkehrung des platonischen Motivs bei Gilles Deleuze und anderen vgl. Dotzler: Simulation, $514 \mathrm{f}$.

45 Hans-Jörg Rheinberger, blog.zhdk.ch/windkanal/tag/windkanal zhdk, gesehen am 18.2.2018. Der Blog dokumentiert die Arbeit mit dem Windkanal auf dem Dach der Zürcher Hochschule der Künste. Siehe dazu auch Florian Dombois (Hg.): The Wind Tunnel Model. Transdisciplinary Encounters, Zürich 2017.

46 Zur kunsthistorischen Perspektive aufWind vgl. Barbara Baert: Pneuma and the Visual Medium in the Middle Ages and Early Modernity, Leuven 2016; zur anthropologischen Perspektive aufWind vgl. Elisabeth Hsu, Chris Low: Wind, Life, Health. Culture, Commerce and Capital, Blackwell 2007.

47 Henning Schmidgen, Peter Geimer, Sven Dierig: Einleitung, in: dies. (Hg.): Kultur im Experiment, Berlin 2004, 7-14, hier 12. Sie beziehen sich hier explizit auf Gilbert Simondon.

\section{Windlabor}

Der Environmental Wind Tunnel vermisst den Raum, den er hervorbringt. Er schreibt sich immer wieder in das ein, was er hilft, aufschreibbar zu machen. Gleichzeitig vermisst er das Aufschreiben selbst. Anders gesagt: Grenzschichtwindkanäle erzeugen keine Daten, sondern Räume, in denen speziell an diese Räume angepasste Messinstrumente Daten erzeugen. Grenzschichtwindkanäle bringen Mikroklimata oder Environments hervor, die dem Einsatz anderer Medientechniken vorausgehen. ${ }^{43}$ Dieses technische Environment oder Mikroklima hakt durch die initiale Kalibrierung in das $\langle$ Feld $>$ oder die $\langle$ Natur $\rangle$ aus der Feldstudie bzw. Naturmessung ein, operiert aber während der Simulation losgelöst von diesen und macht ihre Konstruiertheit dadurch selbst beobachtbar. Der erzeugte Raum ist - im Sinne des (umgekehrten) platonischen Verhältnisses von Sein und Schein - nicht Modell eines Originals, sondern Modell eines Modells. ${ }^{44}$ In Hans-Jörg Rheinbergers Worten formuliert: «Im Grunde ist der Windkanal ein Modell 2. Ordnung. Und gleichzeitig verstörend konkret». ${ }^{45}$ Das indexikalische Verhältnis zwischen atmosphärischer Grenzschicht und Erdoberfläche wird in einem Windkanal in struktureller Analogie wiederholt: Zwischen der technisch erzeugten Atmosphäre und den Oberflächen von Stadtmodell und Windkanal. In der Meteorologie wird die atmosphärische Grenzschicht auch als planetare Grenzschicht oder Peplosphäre bezeichnet (peplos, griech.: Kleid, luftiges Gewand). Im Windkanal wird dieses luftige Gewand einerseits zum technisch erzeugten und kontrollierbaren Environment. Andererseits behält das Kleid eigensinnig etwas von den wehenden Gewändern auf den Gemälden Sandro Botticellis. ${ }^{46}$

Das Fluid Dynamics and Diffusion Laboratory adressierte mit seinen beiden Gewandschneidereien eine Verschiebung der Räumlichkeit des Labors. Wie für andere Labore des 20. Jahrhunderts charakteristisch, veränderte sich auch in diesem der Maßstab des Experimentierens, seine Orte waren «nicht einfach wie Behälter gegeben», sondern die Experimente brachten ihre «Territorien durch ihre Form und Funktionsweise überhaupt erst hervor» und «die dabei entstehenden Umwelten» hingen «vollständig vom Experiment als einer $<$ Existenzbedingung»» ab. ${ }^{47}$ Doch waren diese Verschiebungen weniger einer architektonischen Entgrenzung des Labors geschuldet, einer Expansion, als vielmehr einer zusätzlichen Eingrenzung, einer Art Mise en abyme. In dieser Abimisierung umfassten die Mauern des Laboratoriums nicht nur den ganzen Planeten, ${ }^{48}$ sondern Planet und Laboratorium schachtelten sich in anderer Form ineinander.

Die Faktizität der in diesen Mustern, Verschachtelungen und Spiegelungen produzierten Daten hängt nicht zuletzt davon ab, welcher Erkenntnisanspruch an sie herangetragen wird. An der Schnittstelle von Meteorologie, Ingenieurwissenschaften und Stadtplanung erzeugen diese Daten nämlich kein einheitliches epistemisches Feld; der Grenzschichtwindkanal ist ein Medium zur 
Beobachtung ganz unterschiedlicher Phänomene. So stellen beispielsweise kleine komplexe Wirbel an Oberflächen für Meteorolog_innen ein prekäres epistemisches Objekt dar. Der Grenzschichtwindkanal ist für sie ein Medium, diese beobachtbar zu machen. Für Ingenieur_innen hingegen sind die Luftverwirbelungen vor allem ein Indikator für die Qualität ihrer nachgebildeten Städte und Landschaften sowie ihrer Windkanalkonstruktion. Für sie ist der Grenzschichtwindkanal ein Medium, etwas über ihre Modelle und Instrumente zu erfahren. ${ }^{49}$ Für Stadtplaner_innen, Architekt_innen und Bauingenieur_innen wiederum bedeutet ein Zuviel oder ein Zuwenig an Turbulenz je nach Fragestellung schlichtweg einen Störfaktor. Der Grenzschichtwindkanal ist für sie ein Medium, um die Reibung der von ihnen gestalteten Bauwerke oder Flächen und deren Umgebungen zu minimieren. In zweiter Ordnung ist der Grenzschichtwindkanal zudem ein Medium, um etwas über Beobachtung, Modellierung oder das Verhältnis von Objekten und ihren Umgebungen an sich zu erfahren. All diese verschiedenen Funktionen interferieren insbesondere dann, wenn es um die Frage der Bewertung der Daten geht, die sich je nach den an sie herangetragenen Kriterien zwischen <wahr> und <falsch>, <genau> und <ungenau>, <Signal> und <Rauschen> bewegen. Die analoge Simulation ist eine Wissenspraktik, die in einer für ihren Erkenntnisgewinn konstitutiven Kippposition zwischen diesen Polen situiert ist. Die mit analogen Simulationen in Grenzschichtwindkanälen erzeugten Daten sind Medien einer veränderten Referenz der Signifikanten.

Diese veränderte Referenz wird auch innerhalb der aktuellen stadtmeteorologischen Forschung diskutiert. In einem Aufsatz von 20 I I zu Fragen der Validierung, Verifikation und Repräsentation von Daten heißt es, die Grenzschichtmeteorologie «deals with the well-known fact that data are always to some degree uncertain». ${ }^{50}$ Wegen dieser «uncertainty (better: variability)» sei das Ziel, «data of known quality/uncertainty ${ }^{51} \mathrm{zu}$ generieren. Der «degree of uncertainty ${ }^{52}$ wird in Zahlen beziffert und es wird intensiv an einer internationalen Modellierungsrichtlinie gearbeitet..$^{53}$ Unsicherheit oder Variabilität - bzw. das <verstörend Konkrete>, die wehenden Gewänder Botticellis, der Abgrund der Wiederholungen - sind in Stadtklimasimulationen nicht nur Methode, sondern Standard.

\section{Metutopia}

Wenn Grenzschichtwindkanäle Medientechnologien sind, in denen analoge Simulationen als Gemenge von Praktiken und Akteur_innen <teilweise selbstreferenzielle> Räume erzeugen, dann stellt sich für die Stadtklimaforschung insbesondere die Frage nach dem welterzeugenden Charakter von Simulationen. Die Stadtklimaforschung verzahnt an der Schnittstelle von Meteorologie und Stadtplanung seit dem i9. Jahrhundert wie wenige andere Bereiche Wissenschaft und Politik. Wenn es um Veränderungen der Stadtbegrünung, den Windkomfort für Fußgänger_innen oder die Ausbreitung von Emissionen geht,
48 Vgl. Bruno Latour: Von ‘Tatsachen` zu ‘Sachverhalten`: Wie sollen die neuen kollektiven Experimente protokolliert werden?, in: Schmidgen u.a. (Hg.): Kultur im Experiment, 17-36, hier 19 f. Zudem fand eine Expansion der Begriffe Labor und Experiment für Felder außerhalb der Naturwissenschaften statt. Als Experimente wurden auch Praktiken bezeichnet, die nur noch wenig mit Laboratorien zu tun hatten. Vgl. Philipp Felsch: Das Laboratorium, in: Alexa Geisthövel, Habbo Knoch (Hg.): Orte der Moderne. Erfahrungswelten des 19. und 20. Jahrhunderts, Frankfurt/M., New York 2005, 27-36, hier 3 of.

49 So wurde in den Anfängen der Windkanalforschung häufig diskutiert, ob die Messdaten aus einem Windkanal eher etwas über das Gemessene oder das Messende aussagen.

50 Michael Schatzmann, Bernd Leitl: Issues with validation of urban flow and dispersion CFD models, in: Journal of Wind Engineering and Industrial Aerodynamics, Vol. 99, Nr. 4, April 2011, 169-186, hier 185.

51 Ebd.

52 Ebd., 169.

53 Die Richtlinien werden insbesondere in dem Projekt COST 732 erstellt. Zu diesem und weiteren vgl. Robert Meroney, Ryohji Ohba, Bernd Leitl u. a.: Review of CFD Guidelines for Dispersion Modeling, in: Fluids, Vol. 1, Nr. 14, 2016, 1-16. 
54 Vgl. BMBF (Hg.): Bekanntmachung. Diese zweifache Bedeutung von Veränderung betrifft auch Fragen nach Praktiken der Resilienz oder des Geoengineerings.

55 Helmut Landsberg: The Meteorologically Utopian City, in: Bulletin of the American Meteorological Society, Vol. 54, Nr. 2, 1973, 86-89, hier 86. 56 Ebd.

57 Henri Lefebvre: The Survival of Capitalism. Reproduction of the Relations of Production, New York 1976 [1973], 36 .

58 Friedrich Balke, Bernhard Siegert, Joseph Vogl: Editorial, in: Archiv für Mediengeschichte, Nr. 14: Modelle und Modellierung, 2014, 5-8, hier 6 .

59 Zu möglichen Zukünften durch Computersimulationen vgl. insbesondere Sebastian Vehlken: Plutonium Worlds. Fast Breeders, Systems Analysis and Computer Simulation in the Age of Hypotheticality, in: communication+1, Vol. 3: Afterlives of Systems, 2014, Article 7.

60 Vgl. Robert Meroney, Tetsuji Yamada: Wind Tunnel and Numerical Experiments of Two Dimensional Stratified Airflow over a Heated Island, Abschlussbericht Fluid Dynamics and Diffusion Laboratory, Fort Collins 1971, 7. Diese wird als Pionierstudie auf dem Gebiet des Computational Wind Engineering angesehen, vgl. Bernd Blocken: 50 Years of Computational Wind Engineering. Past, Present and Future, in: Journal of Wind Engineering and Industrial Aerodynamics, Vol. ${ }^{169}$, 2014, 69-102, hier 70. werden Grenzschichtwindkanäle zu Medien der Entscheidung und Planung. Der Titel der eingangs vorgestellten BMBF-Fördermaßnahme [UC]2 - Urban Climate Under Change ist insofern durchaus Programm: «Under Change» adressiert Städte gleichermaßen als Betroffene und Verursacher_innen von Klimawandel. So würden urbane Gebiete einerseits hochsensibel auf Veränderungen des Klimas wie ausgedehnte Hitzeperioden, starke Unwetter oder Hochwasser reagieren; andererseits seien sie für bis zu $70 \%$ des TreibhausgasAusstoßes der Menschheit verantwortlich. ${ }^{54}$ Sie sind damit dezidiert nicht nur Untersuchungsobjekte der Klimawandelforschung, sondern auch der Klimafolgenforschung. Nicht umsonst ist das Förderprogramm des BMBF Teil einer BMBF-Leitinitiative mit dem Titel Zukunftsstadt.

I973, zwei Jahre nach den ersten Tests im Environmental Wind Tunnel, proklamierte der Meteorologe Helmut Landsberg in seinem Aufsatz «The Meteorologically Utopian City» die Notwendigkeit der Beteiligung von Meteorolog_innen in der Stadtplanung. Landsberg, I969 bis 1978 Vorsitzender der Kommission für Klimatologie der World Meteorological Organization (WMO) der Vereinten Nationen, prognostizierte angesichts wachsender Städte und zunehmender Urbanisierung: «In the meteorologically utopian city [...] - let me call it Metutopia - [...] the role of the meteorologist as a forecaster and controller of urban pollution will be a central one».55 In der Überlagerung von Stadtplanung und Meteorologie verschiebt sich die Utopie zur Metutopie und damit von einem Gesellschaftsentwurf, der nie Realität werden wird, zu einem, der durchaus umsetzbar klingt, wenn Landsberg beschreibt, dass Meteorolog_innen aus Fakten ein optimales Design erarbeiten und dieses mit anderen Realitäten, «other realities», abgleichen sollen. ${ }^{56} \mathrm{Im}$ gleichen Jahr wie Landsberg führt der Soziologe und Philosoph Henri Lefebvre seine ähnlich machbar konzipierte urbane Utopie näher aus, die er I 968 in seinem Essay «Right on Cities» entworfen hatte und in der die Vorstellung des Unvorstellbaren Konzepte für ein Morgen generiert: «In order to extend the possible, it is necessary to proclaim and desire the impossible. Action and strategy consist in making possible tomorrow what is impossible today». ${ }^{57}$ Stadtklimasimulationen ab Anfang der I97oer Jahre figurieren vor diesem Hintergrund nicht nur in Landsbergs Sinne als Techniken der Vorhersage und Kontrolle, sondern mit Lefebvres Worten auch als Aktionen und Strategien der Ermöglichung des noch Unmöglichen. In medienwissenschaftlicher Perspektive ist herausgearbeitet worden, dass Modelle sich «in einer Grauzone zwischen Faktizität und Fiktion» bewegen und gerade deswegen «einen wesentlichen Beitrag für die Erprobung vergangener oder künftiger Realitäten ${ }^{58}$ leisten können. Sie erlauben, in anderen Worten, das Durchspielen verschiedener Szenarien und ermöglichen oder verhindern diese dadurch. ${ }^{59}$

Im Fluid Dynamics and Diffusion Laboratory wird I97 I nicht nur das erste Mal versucht, den urbanen Hitzeinseleffekt in einem Grenzschichtwindkanal zu simulieren. Parallel wird auch an einer numerischen One-to-one-Simulation 
im Computer gearbeitet, welche die im Windkanal auftretenden Skalierungsschwierigkeiten umgehen und mehrere Windkanalexperimente miteinander verbinden soll. ${ }^{60} \ll$ Once confidence is established in numerical procedures through a wind tunnel simulation», so heißt es in dem Abschlussbericht zu diesem numerischen Versuch, «the direct application of the numerical program to the atmosphere is reasonable». Der Grenzschichtwindkanal wurde nicht nur als Werkzeug zur Verarbeitung und Erzeugung von Daten und als Medium der Entscheidung und Planung verwendet, sondern auch als Werkzeug der Validierung. Die aktuell zunehmende Simulierbarkeit von Stadtklima mit Hochleistungsrechnern verfestigt diese Aufgabe: Grenzschichtwindkanäle werden zur Überprüfung der mit Computersimulationen erzeugten Daten eingesetzt ebenso wie zur Erzeugung von Daten für diese. ${ }^{61}$ Die verbesserte numerische Modellierung ist für die Stadtklimaforschung nicht nur wissenschaftlich, sondern auch politisch zentral. So spielten Städte und der mikrometeorologische Bereich im Weltklimarat, dem IPCC - Intergovernmental Panel on Climate Change, vorerst keine Rolle, der Fokus lag auf globalen Klimavorhersagen. Auch im Kyotoprotokoll wurden Städte nicht erwähnt. ${ }^{62}$ Die weltpolitische Aufmerksamkeit für Städte in Klimadebatten, so konstatiert die historisch arbeitende Stadtklimaforschung, habe erst in den letzten zehn Jahren zugenommen. Dies liege nicht zuletzt daran, dass die Stadt als Environment zunehmend in numerischen Wetter- und Klimamodellen aufgelöst werden könne. ${ }^{63}$ Erste funktionsfähige Computersimulationen von Städten und ihrem Klima erlauben es, dass Städte innerhalb des globalen Zirkulationssystems überhaupt sichtbar werden.
61 Wegen dieses Status werden sie in der Meteorologie auch als «Mediatoren zwischen Feldmessungen und Computerexperimenten bezeichnet.

62 Vgl. Michael Hebbert, Vladimir Jankovic: Cities and Climate Change. The Precedents and Why They Matter, in: Urban Studies, Vol. 50, Nr. 7 , Mai 2013, 1332-1347, hier 1332.

63 Vgl. ebd., 1333. Die geplante Stadtklimasimulation des BMBF soll zudem «die Verzahnung von Daten zu Klimaveränderungen mit demographischen und sozialen bzw. gesellschaftlichen Daten» erlauben, vgl. BMBF (Hg.): Bekanntmachung, B4. Isabell Schrickel und Christoph Engemann weisen darauf hin, dass eine der Stärken von Computersimulationen darin bestehe, dass diese "questions of scale and scalability and hence about the interdependence and mobility of phenomena between local and global scales» adressieren würden, vgl. dies.: Trading Zones of Climate Change. Introduction, in: Berichte zur Wissenschaftsgeschichte, Vol. 40, Nr. 2, Juni 2017, 111-119, hier 112. 


\author{
BERNHARD PÖRKSEN Und CLAUS PIAS \\ vorgestellt von EVA SCHAUERTE und SEBASTIAN VEHLKEN
}

\title{
DEBATTE ÜBER WIDERSPENSTIGE WAHRHEITEN
}

Wir haben Bernhard Pörksen als Autor angefragt, weil er eine ausgewiesene Stimme in der aktuellen gesellschaftlichen Debatte um Faktizität darstellt. Sowohl in seinen wissenschaftlichen Publikationen als auch im politischen und feuilletonistischen Kontext setzt er sich mit Kontroversen um Fake News oder alternative Fakten auseinander. Ebenso antwortet er auf eine Polemik gegenüber postmodernen Philosophiemodellen und wendet sich dezidiert gegen den Vorwurf, der Konstruktivismus habe maßgeblich zur Sinnentleerung des gegenwärtigen Diskurses beigetragen und die Geburt ‘alternativer Fakten` befördert. Dabei gibt Pörksen einer über akademische Binnenbezüge hinausweisenden Positionierung den Vorzug vor einer klassisch-distanzierten, kulturwissenschaftlichen Analyse, auch um die Tür zu einer Debatte aufzustoßen, die nicht nur im Kontext akademischer Diskurskultur stattfinden sollte.

Der Kommentar von Claus Pias bezieht sich vor allem auf eine von Pörksen angerissene größere Debatte. Diese betrifft den Horizont einer möglichen Kritik an Veränderungen, die durch digitale Kulturen angestoßen werden und von denen die in diesem Schwerpunkt behandelte Frage nach Faktizitäten nur eine ist. Pias thematisiert erstens einen blinden Fleck gegenüber der Geschichte von Medientheoriebildung selbst. Zweitens greift er Pörksens Kritik an akademischen Diskussionskulturen auf, verbindet sie mit einem derzeitigen Diskurs der Universitätskritik und appelliert an eine historische Aufarbeitung der Postmoderne. Und drittens erweitert er die Debatte um Faktizitäten mit einer Reihe von Hinweisen auf Kritikpotenziale, die sich jenseits des Begriffsrepertoires moderner Konzeptualisierungen von Fake und Fakt bewegen und ihre "Gegenbegriffe» in vormodernen Kontexten zu suchen.

Wir laden interessierte Leser_innen ein, die Diskussion aufder Website der ZfM weiter zu führen.

zfmedienwissenschaft.de/online/debatte/widerspenstige-wahrheiten 